Vietnam Academy of Science and Technology
Vietnam Journal of Earth Sciences
http://www.vjs.ac.vn/index.php/jse

\title{
Prediction of maximum earthquake magnitude for northern Vietnam region based on the gev distribution
}

Vu Thi Hoan ${ }^{* 1}$, Ngo Thi Lu${ }^{1}$, Mikhail Rodkin², Nguyen Huu Tuyen¹, Phung Thi Thu Hang', Tran Viet Phuong ${ }^{1}$

${ }^{1}$ Institute of Geophysics, Vietnam Academy of Science and Technology

${ }^{2}$ International Institute of Earthquakes Prediction Theory and Mathematical Geophysics, RAS, Moscow

Received 1 March 2016. Accepted 15 December 2016

\begin{abstract}
The present work is a continuation and improvement of the application of the generalized extreme value distribution to study the seismicity of the Southeast Asia. We have applied the generalized extreme value distribution (GEV) method to estimate maximum magnitude value $\left(\mathrm{M}_{\max }\right)$ for the earthquake catalog of Northern Vietnam. Using this method, we obtain the distribution of maximum earthquake magnitude values. This distribution can be characterized by its quantile $\mathrm{Q}_{\mathrm{q}}(\tau)$ at any desirable statistical level $\mathrm{q}$. The quantile $\mathrm{Q}_{\mathrm{q}}(\tau)$ provides a much more stable and robust characteristic than the traditional absolute maximum magnitude $\mathrm{M}_{\max }\left(\mathrm{M}_{\max }\right.$ can be obtained as the limit of $\mathrm{Q}_{\mathrm{q}}(\tau)$ as $\left.\mathrm{q} \rightarrow 1, \tau \rightarrow \infty\right)$. The parameters have been obtained: $\zeta=-0.178 \pm 0.08 ; \sigma=0.23 \pm 0.08 ; \mu=4.39 \pm 0.16$; $\mathrm{M}_{\max }=6.8$ with the probability of $98 \%$ for period $2014-2064$.
\end{abstract}

Keywords: Maximum magnitude $\left(\mathrm{M}_{\max }\right)$, generalized extreme value distribution (GEV), earthquake prediction, seismic hazard.

(C2016 Vietnam Academy of Science and Technology

\section{Introduction}

The NorthernVietnam region is the most active tectonic and high potential risk area of Vietnam. The parameter $\mathbf{M}_{\max }$ represents the maximum of possible earthquake magnitude in the study region. This parameter plays a very important role in seismic hazard assessment and mitigation of the seismic risk. Giving a reliable estimate of $\mathbf{M}_{\max }$, it is comparatively easy to take adequate decisions on the construction standards of buildings or

*Corresponding author, Email: hoanvt84@gmail.com on the insurance policy (Pisarenko et al., 2014b). Therefore, the maximum magnitude earthquake prediction is not only the task with the scientific sense but also an imperative task for the seismic practice of Vietnam.

There are many methods to assess maximum earthquake magnitude including the geological extrapolation (Phan et al., 2012, 2013), calculation of $M_{\max }$ base on size of earthquake source zone (Nguyen N.T et al., 2005; Bui et al., 2013), probabilistic methods... (Gumbel, 1958; Nguyen H.P, 1991, Nguyen N.T et al., 2005, Nguyen H.P et al., 
Vu Thi Hoan, et al./Vietnam Journal of Earth Sciences 38 (2016)

1997, 2001, 2014). One of the probabilistic methods is based on the generalized extreme value distribution $(G E V)$. This method is introduced by Pisarenko et al. for the Harvard catalog (Pisarenko et al., 2007, 2008), the catalogs of Japan (Pisarenko et al., 2010) and Vietnam (Pisarenko et al., 2012). We used this method to assess $\mathbf{M}_{\max }$ for Southeast Asia and obtained $M_{\max }^{\text {Predict }}=8,235$ for period 2013 2063 with probability $98 \%$ (Vu et al., 2014). In this work we continue to use this method to assess $\mathbf{M}_{\max }$ for the Northern Vietnam and obtained $M_{\max }^{\text {Predict }}=6,8$ for period $2014-2064$ with probability $98 \%$.

\section{Methodology and used data}

\subsection{Used data}

The study area is limited by the coordinates $\varphi=17^{\circ} \div 24^{\circ} \mathrm{N} ; \lambda=102^{\circ} \div 110^{\circ} \mathrm{E}$ (Figure 1).

We collect data from various sources: the Department of the seismological survey, the Earthquake Information and Tsunami Warning Centre, the previously published earthquake catalog on the territory of Vietnam and the data from International Seismological
Center - ISC. In the data from ISC, an earthquake can have 4 types of magnitude: Local magnitude $\left(\mathrm{M}_{\mathrm{L}}\right)$, body - wave magnitude $\left(\mathrm{m}_{\mathrm{b}}\right)$, surface - wave magnitude $\left(\mathrm{M}_{\mathrm{s}}\right)$, moment magnitude $\left(\mathrm{M}_{\mathrm{w}}\right)$. However, as $\mathrm{M}_{\mathrm{L}}$ is the most common magnitude used in Vietnam, the $M_{L}$ values were chosen for the entire catalog. It is possible to convert $\mathrm{m}_{\mathrm{b}}, \mathrm{M}_{\mathrm{s}}$, $\mathrm{M}_{\mathrm{w}}$ values to $\mathrm{M}_{\mathrm{L}}$. The collected data have 1376 earthquakes with magnitudes $\mathrm{M}=1.7-7.5$.

After separation of foreshocks and aftershocks from this earthquake catalog, we get independent earthquake catalog including 1196 independent earthquakes with magnitude $1.7 \leq \mathrm{M} \leq 7.5$ for Northern Vietnam and surrounding regions.

The data in this catalog are continuous on time since 1972, so we chose the period from 1972 to 2014 for estimation of $\mathrm{M}_{\max }$. There are 349 earthquakes with $\mathrm{M} \geq 4.1$ in the period.

\subsection{Prediction method}

The distribution function generalized extreme value is defined as follows (Pisarenko et al., 2007, 2008, 2010):

$$
\begin{aligned}
& \operatorname{GEV}(\mathrm{x} \mid \sigma, \mu, \zeta) \\
& =\left\{\begin{array}{r}
\exp (-(1+(\zeta / \sigma) \cdot(\mathrm{x}-\mu))-1 / \zeta, \zeta<0 ; \sigma>0 ; x \leq \mu-\sigma / \zeta, \zeta \neq 0 \\
\exp \left(-\exp \left[-\frac{\mathrm{x}-\mu}{\sigma}\right]\right), \quad \zeta=0
\end{array}\right.
\end{aligned}
$$

Where $\mathrm{x}$ is variable representing the magnitude earthquake value, $\sigma$ is the scale parameter, $\mu$ is the location parameter, $\zeta$ is the form parameter.

To determine the GEV function we need to identify 3 parameters $\zeta, \sigma, \mu$ in formula (1). These parameters $\zeta, \sigma, \mu$ are determined in

$$
\frac{1}{n} \sum_{k=1}^{n}\left(x_{k}-M 1\right)^{3}=(\sigma)^{3}\left[-2(\Gamma(-\zeta))^{3}-\frac{6}{\zeta} \Gamma(-\zeta) \Gamma(-2 \zeta)-\frac{3}{\zeta^{2}} \Gamma(-3 \zeta)\right]=M 3
$$

where $\Gamma(\mathrm{x})$ is the Gamma function: $\Gamma(\mathrm{t})$ $=\int_{0}^{\infty} x^{t-1} e^{-x} d x, \mathrm{n}$ is the number of earthquakes in each T-intervals, $\mathrm{x}_{\mathrm{k}}$ is magnitude of $k^{\text {th }}$ earthquake. each period $\mathrm{T}$, by solving the set $\mathrm{o}$ : three equations below:

$$
\frac{1}{n} \sum_{k=1}^{n} x_{k}=\mu-\frac{\sigma}{\zeta}+\frac{\sigma}{\zeta} \Gamma(1-\zeta)=M 1
$$

$$
\begin{aligned}
& \frac{1}{n} \sum_{k=1}^{n}\left(x_{k}-M 1\right)^{2}=\left(\frac{\sigma}{\zeta}\right)^{2}[\Gamma(1-2 \zeta)- \\
& \left.(\Gamma(1-\zeta))^{2}\right]=M 2
\end{aligned}
$$

It is important to determine T-intervals to suit each catalog because T-intervals have the influence on the values of the three parameters $\zeta, \sigma, \mu$ of the GEV function. To 
find T-intervals, we need to determine the density Poisson distribution ( $\lambda$ ) of the magnitude earthquake values:

$\lambda=\frac{N}{t}$, where $\mathrm{N}$ is the number of independent earthquakes, $t$ is the time between the first event and the last event.

The chosen T-values (days) must satisfy three conditions:

All T-intervals are non-empty.

Value $1 / \lambda T \rightarrow 0$ (with $\lambda$ is the frequency earthquakes with magnitude $M \geq m$ ).

Value of parameter $\zeta$ is stable enough to determine the GEV function.

The following steps should be taken:

- Choose an interval of values $\left(\mathrm{T}_{\mathrm{L}} ; \mathrm{T}_{\mathrm{H}}\right)$ for time interval durations $\mathrm{T}$, for which the catalog still contains a sufficient number of $\mathrm{T}$ intervals (with $\mathrm{T}_{\mathrm{L}}$ is the lowest time; $\mathrm{T}_{\mathrm{H}}$ is the highest time);

- Choose in this interval $\left(\mathrm{T}_{\mathrm{L}} ; \mathrm{T}_{\mathrm{H}}\right)$ a finite set of $\mathrm{u}$ time-interval durations $\mathrm{T}$ $\left(\mathrm{T}_{\mathrm{L}} \leq \mathrm{T}_{1}<\mathrm{T}_{2}<\ldots<\mathrm{T}_{\mathrm{u}} \leq \mathrm{T}_{\mathrm{H}}\right)$;

- The GEV parameters are estimated by the method of moments (Pisarenko et al., 2007, $2008,2010)$ for each of the u time - interval durations $\mathrm{T}$, which yields the following set of parameters:

$\zeta\left(\mathrm{T}_{1}\right), \zeta\left(\mathrm{T}_{2}\right), \ldots, \zeta\left(\mathrm{T}_{\mathrm{u}}\right), \sigma\left(\mathrm{T}_{1}\right), \sigma\left(\mathrm{T}_{2}\right), \ldots$, $\sigma\left(\mathrm{T}_{\mathrm{u}}\right), \mu\left(\mathrm{T}_{1}\right), \mu\left(\mathrm{T}_{2}\right), \ldots, \mu\left(\mathrm{T}_{\mathrm{u}}\right)$;

- To estimate the average values $\bar{\zeta}, \bar{\sigma}$, $\mu$ of the GEV parameters $\zeta, \sigma, \mu$

- The $\tau$ is the predicted period (from the time of the earthquake event was chosen as supporting event). The parameters $\zeta, \sigma, \mu$ are represented as the functions of $\tau$ by the formulas (5-7) below:

$$
\zeta(\tau)=\zeta(\mathrm{T})
$$

$$
\begin{aligned}
& \quad \sigma(\tau)=\sigma(\mathrm{T}) \cdot(\tau / \mathrm{T})^{\xi} ; \\
& \mu(\tau)=\mu(\mathrm{T})+(\sigma(\mathrm{T}) / \xi) \cdot\left((\tau / \mathrm{T})^{\xi}-1\right) ; \\
& \text { - The quantile in this period is: } \\
& \mathrm{Q}_{\mathrm{q}}(\tau)=\mathrm{h}+(\mathrm{s} / \xi) \cdot\left(\mathrm{a} \cdot(\lambda \tau)^{\xi}-1\right) \\
& \text { where: } \\
& \mathrm{a}=(\log (1 / \mathrm{q}))^{-\xi}, \\
& \mathrm{h}=\mu+(\sigma / \xi) \cdot\left((\lambda \mathrm{T})^{-\xi}-1 ;\right. \\
& \mathrm{s}=\sigma \cdot(\lambda \mathrm{T})^{-\xi} \text {. } \\
& \text { When } \tau \rightarrow \infty \text { then } \mathrm{Q}_{\mathrm{q}}(\tau)=\mathrm{M}_{\max }(\tau) \rightarrow \mathrm{M}_{\max }: \\
& \mathrm{M}_{\text {max }} \text { predict }=\lim _{\tau \rightarrow \infty} \mathrm{Q}_{\mathrm{q}}(\tau)
\end{aligned}
$$

Thus, after finding the appropriate $\mathrm{T}$ intervals, three parameters $\zeta, \sigma, \mu$ can be found in each time period. The obtained results can be used to determine the content of $\mathrm{GEV}$, decile point value of $\mathrm{Q}_{\mathrm{q}}(\tau)$, and to assess the $\mathrm{M}_{\max }$ value.

\section{Calculation results}

In this section, we present the calculation results for the given data set.

Step 1: Calculate the density Poisson distribution $(\lambda)$

The period from 23/1/1972 $\left(\mathrm{t}_{1}\right)$ to $20 / 8 / 2014\left(t_{n}\right)$ used with the daily unit. The total time are 15518.71 days. The number of T-intervals is $\mathrm{n}: \mathrm{n}=$ interger $\frac{t_{n-t_{1}}}{T}$

\begin{tabular}{|c|c|c|c|c|c|c|c|c|c|c|c|c|}
\hline & 255 & 265 & 275 & 285 & 295 & 305 & 315 & 325 & 335 & 345 & 355 & 365 \\
\hline & .735 & .96 & 18 & .410 & .6 & 5.8 & 0 & 7.3 & 5 & 7.7 & 7.984 & 3.2 \\
\hline$\lambda T$ & 0.174 & 0.168 & 0.162 & 0.156 & 0.151 & 0.146 & 0.141 & 0.137 & 0.133 & 0.129 & 0.125 & 0.122 \\
\hline
\end{tabular}

Density $\lambda$ Poisson distribution is calculated as follows:

$$
\lambda=\frac{N}{t}=\frac{349}{15518.71}=0.02249
$$

\section{Step 2: Select the jump (T)}

According to the data in the catalog, to satisfy the condition (a) above, the smallest value of $\mathrm{T}$-intervals is 250 days. The $\mathrm{T}$ intervals in the corresponding product $\lambda \mathrm{T}$ are the following:

Table 1. The parameters $\mathrm{T}, \lambda \mathrm{T}, 1 / \lambda \mathrm{T}$

From this table, the greater T-intervals are, the smaller value of the ratios $(1 / \lambda \mathrm{T})$ are. In principle, the closer values $(1 / \lambda \mathrm{T})$ are to the value " 0 ", the better T-intervals are. However, to satisfy the condition (c), Figure 2 shows an approximate "stabilization" of the $\zeta$ - estimates 
Vu Thi Hoan, et al./Vietnam Journal of Earth Sciences 38 (2016)

in the range 300 and 350 days. Therefore, to satisfy the above conditions, the value of $\mathrm{T}$ interval is 350 days. With $\mathrm{T}=\mathbf{3 5 0}$ days, then

$$
\mathrm{n}=\text { interger } \frac{t_{n-t_{1}}}{T}=44 \text {. }
$$

Step 3: Determine the parameters $\zeta, \sigma, \mu$

In each $\mathrm{u}$ time-interval durations $\mathrm{T}\left(\mathrm{T}_{\mathrm{L}} \leq\right.$ $\mathrm{T}_{1}<\mathrm{T}_{2}<\ldots<\mathrm{T}_{\mathrm{u}} \leq \mathrm{T}_{\mathrm{H}}$ ), the parameters $\zeta, \sigma, \mu$ are determined in each period $\mathrm{T}$, by solving the set of three equations (2-4).

$\zeta\left(\mathrm{T}_{1}\right), \zeta\left(\mathrm{T}_{2}\right), \ldots, \zeta\left(\mathrm{T}_{\mathrm{u}}\right), \sigma\left(\mathrm{T}_{1}\right), \sigma\left(\mathrm{T}_{2}\right), \ldots$, $\sigma\left(\mathrm{T}_{\mathrm{u}}\right), \mu\left(\mathrm{T}_{1}\right), \mu\left(\mathrm{T}_{2}\right), \ldots, \mu\left(\mathrm{T}_{\mathrm{u}}\right)$;

To estimate the average these values:

$$
\bar{\zeta}=-0.178 ; \bar{\sigma}=0.23 ; \bar{\mu}=4.39 \text {. }
$$

In order to estimate the Mean Square Error (MSE) of these estimates, we use formulas (Pisarenko et al., 2008):

$$
\begin{aligned}
M E S_{\zeta} & =\left[(1 / n) \sum_{j=1}^{n}\left(\zeta_{\mathrm{j}}-\bar{\zeta}\right)^{2}\right]^{1 / 2} \\
\operatorname{MES}_{\sigma} & =\left[(1 / n) \sum_{j=1}^{n}\left(\sigma_{\mathrm{j}}-\bar{\sigma}\right)^{2}\right]^{1 / 2} \\
\operatorname{MES}_{\mu} & =\left[(1 / n) \sum_{j=1}^{n}\left(\mu_{\mathrm{j}}-\bar{\mu}\right)^{2}\right]^{1 / 2}
\end{aligned}
$$

Therefore, the parameters are:

$\zeta=-0.178 \pm 0.08 ; \sigma=0.23 \pm 0.08 ; \mu=4.39$ \pm 0.16 .

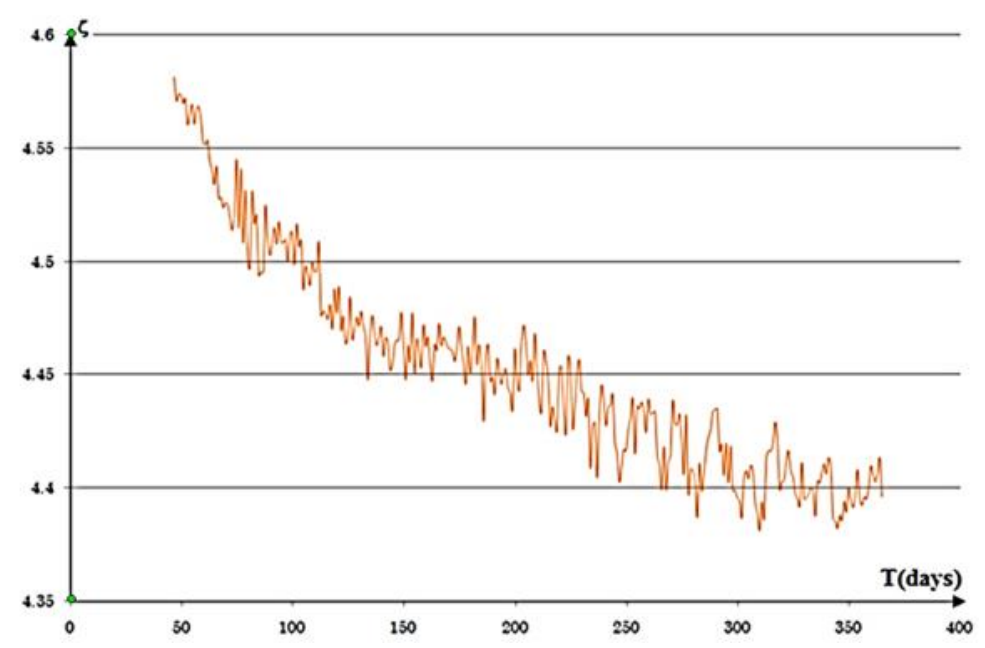

Figure 2. Graph of the $\zeta(\mathrm{T})$ function

Step 4: Determine predicted $M_{\max }$

In the earthquake catalog used, the last strogest earthquake, which occurred 29.06.2014 with magnitude $M=4.4$, has satisfied above specified conditions. So we have chosen this event as supporting event.

$$
M_{\text {max }}^{\text {Predict }}=\lim _{\tau \rightarrow \infty} \mathrm{Qq}(\tau)
$$

\begin{tabular}{|c|c|c|c|c|c|}
\hline$\tau$ (years) & 10 & 20 & 30 & 40 & 50 \\
\hline Period & $2014-2024$ & $2014-2034$ & 2014-2044 & 2014-2054 & $2014-2064$ \\
\hline 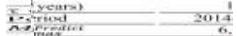 & 6,67 & 6,72 & 6,75 & 6,78 & 6,80 \\
\hline
\end{tabular}

With predicted probability $98 \%$, we get the graph of the function $Q_{q}(\tau)$ in Figure 3 .

From figure 3, we have:

$$
\begin{aligned}
& M_{\text {max }}^{\text {Predict }}=\lim _{\tau \rightarrow 10} \mathrm{Qq}(\tau)=6,67 ; \\
& M_{\text {max }}^{\text {Pred }}=\lim _{\tau \rightarrow 20} \mathrm{Qq}(\tau)=6,72 ; \\
& M_{\text {max }}^{\text {Predict }}=\lim _{\tau \rightarrow 30} \mathrm{Qq}(\tau)=6,75 ; \\
& M_{\text {max }}^{\text {Predict }}=\lim _{\tau \rightarrow 40} \mathrm{Qq}(\tau)=6,78 ; \\
& M_{\text {max }}^{\text {Predict }}=\lim _{\tau \rightarrow 50} \mathrm{Qq}(\tau)=6,8
\end{aligned}
$$




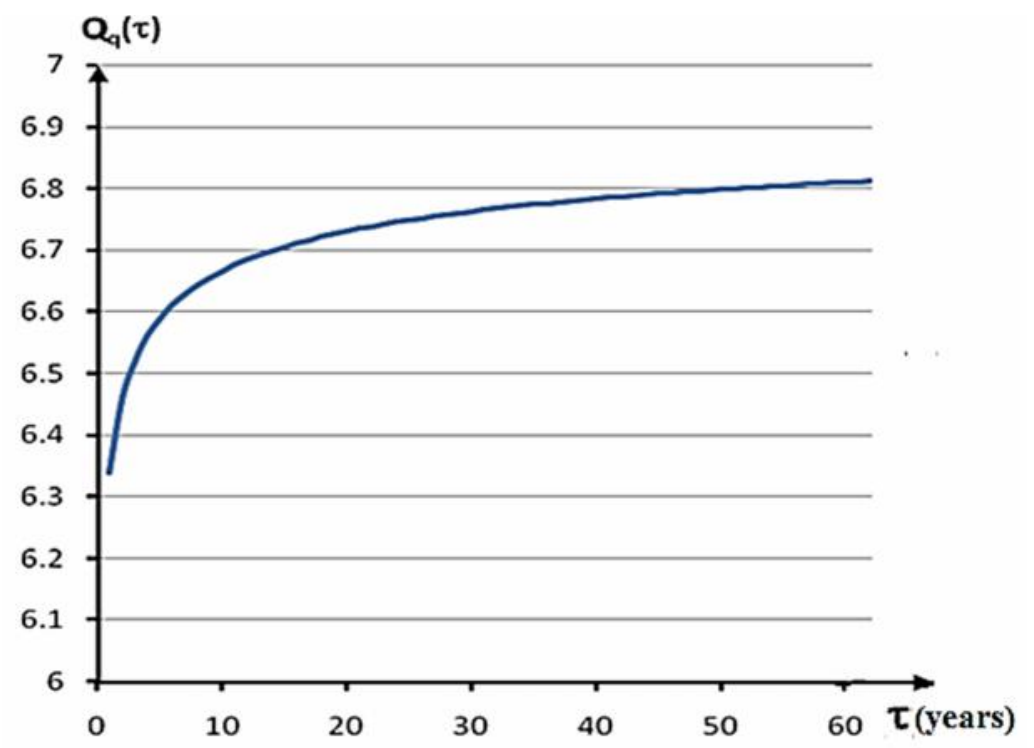

Figure 3. Graph of the $\mathrm{Q}_{\mathrm{q}}(\tau)$ function with $\mathrm{q}=0.98$ for the Northern Vietnam

\section{Discussions}

Largest earthquake is predicted to occur in the Northern Vietnam by GEV method is $M_{\max }^{\text {Predict }}=6.8$ in the next 50 years. This result is quite consistent with the results obtained in the work (Nguyen Ngoc Thuy, 2005), but there are differences compared to the results in the works (Cao Dinh Trong, 2013) $\left(\mathrm{M}_{\max }=6.7\right)$, (Ngo Thi Lu, 2012) $\left(\mathrm{M}_{\max }\right.$ = 7.0); (Nguyen Hong Phuong 1991) $\left(\mathrm{M}_{\max }=\right.$ 7.0); Phan Trong Trinh et al., 2012) $\left(\mathrm{M}_{\max }=\right.$ 7.0); Pham Van Thuc and Kijko $\left(\mathrm{M}_{\max }=7.2\right)$; (Nguyen Hong Phuong, 1997) $\left(\mathrm{M}_{\max }=7.3\right)$. Such differences may be due to the different studied zones, the methods used and the limitations of the length of data period considered (only in 42 years (19722014)).

\section{Conclusions}

On the basis of the catalog of independent earthquakes in period 1972-2014, the maximum earthquake magnitude value was assessed for the Northern Vietnam using GEV method.
We obtained the following sample estimates for this catalog with $\mathrm{T}=350$ days: $\zeta=-0.178 \pm 0.08 ; \sigma=0.23 \pm 0.08 ; \mu=4.39$ $\pm 0.16$

This distribution can be characterized by its quantile $Q_{q}(\tau)$ at any desirable statistical level $q$. With predicted probability $98 \%$, we obtained $M_{\text {max }}^{\text {Predict }}=\lim _{\tau \rightarrow \alpha} \mathrm{Qq}(\tau)=6.8$ for period 2014 - 2064.

The authors would like to thank for the grants from the project research code VAST.ĐL. 01 /14-16: "Development of a set of programs for earthquake prediction by combinations of the statistical, seismic, geophysical and geomorphological methods, and application to the Northwest region of Vietnam" and the project research code VAST.HTQT.NGA.08/15-16: "An approach of the natural phenomena analysis and computer performance for seismogenic assessment of Vietnam territory".

\section{References}

Bui Van Duan, Nguyen Cong Thang, Nguyen Van Vuong, Pham Dinh Nguyen, 2013. The magnitude of the largest possible earthquake in the Muong $\mathrm{La}$ Bac Yen fault zone. Journal of Sciences of the Earth, 35(1), 49-53. 


\section{Vu Thi Hoan, et al./Vietnam Journal of Earth Sciences 38 (2016)}

Cao Dinh Trong, Le Van Dung, Pham Nam Hung, Mai Xuan Bach, 2013. The neural network method for intermediate earthquake prediction (example in Vietnam). Journal of Marine Science and Technology, 3A(13), 17-24.

Gumbel E. J, 1958. Statistics of Extremes, Columbia Univ. Press.

Ngo Thi Lu, Tran Viet Phuong, 2012. About the approach to building algorithms and processes to predict earthquakes by statistical model. Journal of Sciences of the Earth, 34(4), 535-541.

Ngo Thi Lu, Tran Viet Phuong, 2013. Building a new algorithm of the program for separation of forshock and aftershock groups from earthquake catalog to ensure the independence of the events. Journal of Marine Science and Technology. 3A(13), 79-85.

Ngo Thi Lu, Tran Viet Phuong, 2016a. Prediction earthquake (time, location and magnitude of an earthquake) for the Northwest region of Vietnam by program's VAST. ĐL.01/14-16. Thematic reports, 14 pages.

Nguyen Hong Phuong, 1991. Probabilistic assessment of earthquake hazard in Vietnam based on seismotectonic regionalization. Tectonophysics, 198, 81-93.

Nguyen Hong Phuong, 1997. Estimation of maximum earthquake magnitudes for seismic source zones of Vietnam using probabilistic methods. Contributions of marine geology and geophysics, 3, 48-65. Science and technics publishing house. Hanoi.

Nguyen Hong Phuong, 2001. Probabilistic Seismic Hazard Assessment Along the Southeastern Coast of Vietnam, Natural Hazards 24, 53-74.

Nguyen Hong Phuong, Pham The Truyen, 2014. Probabilistic Seismic Hazard Assessment for the South Central Vietnam. Vietnam J. Earth Sci., 36(4), 451-461.

Nguyen Ngoc Thuy, 2005. Project report of KC. 08. 10: "Zoning detailed forecast earthquake in the Northwest of Vietnam", 547p.

Phan Trong Trinh, Ngo Van Liem, Nguyen Van Huong, Hoang Quang Vinh, Bui Van Thom, Bui Thi Thao, Mai Thanh Tan, Nguyen Hoang, 2012. Late Quaternary tectonics and seismotectonics along the Red River fault zone, North Vietnam. Earth-Science Reviews, 114, 224-235.

Phan Trong Trinh, Hoang Quang Vinh, Nguyen Van Huong, Ngo Van Liem, 2013. Active fault segmentation and seismic hazard in Hoa Binh reservoir, Vietnam. Cent. Eur. J. Geosci. 5(2), 223-235.

Pham Van Thuc and Kijko, A., 1985. Estimation of maximum magnitude and seismic hazard in Southeast Asia and Vietnam. Acta Geophys. Pol., XXX111(4), 377-387.

Pisarenko V.F and Rodkin M.V, 2007. Distributions with Heavy Tails: Application to the Analysis of catastrophes, Coputational Seismology issue 38, $242 \mathrm{p}$.

Pisarenko V.F, Sornette A, Sornette D and Rodkin M.V, 2008. New approach to the Characterization of $M_{\max }$ and of the Tail of the Distribution of Earthquake Magnitudes. Pure and Applied Geophysics, 165, 847-888.

Pisarenko V.F, Sornette D and Rodkin M.V, 2010. Distribution of maximum Earthquake magnitudes in future time intervals: application to the seismicity of Japan (1923-2007). EPS (Earth, Planets and Space), 62, 567-578.

Pisarenko V.F, Rodkin M.V, Ngo Thi Lu, 2012. New general quantile approach to the seismic risk assessment application to the Vietnam region. Proceedings of the international scientific conference, 161-167.

Pisarenko V.F, Rodkin M.V, and Rukavishnikova T. A, 2014a. Estimation of the Probability of Strongest Seismic Disasters Based on the Extreme Value Theory. Physics of the Solid Earth, 50(3), 311-324.

Pisarenko V.F, Sornette A, Sornette D and Rodkin M.V, 2014b. Characterization of the Tail of the Distribution of Earthquake Magnitudes by Combining the GEV and GPD Descriptions of Extreme Value Theory. Pure Appl. Geophys. 171, 1599-1624.

$\mathrm{Vu}$ Thi Hoan, Ngo Th Lu, M.V. Rodkin, Tran Viet Phuong, 2014. Application of the generalized extreme value distribution to study the seismicity of the Southeast Asian. Journal of Geology. Series A, 2014, 341-345. Hanoi, 180-189.

Http://www.isc.ac.uk/iscbulletin/search/bulletin/ 